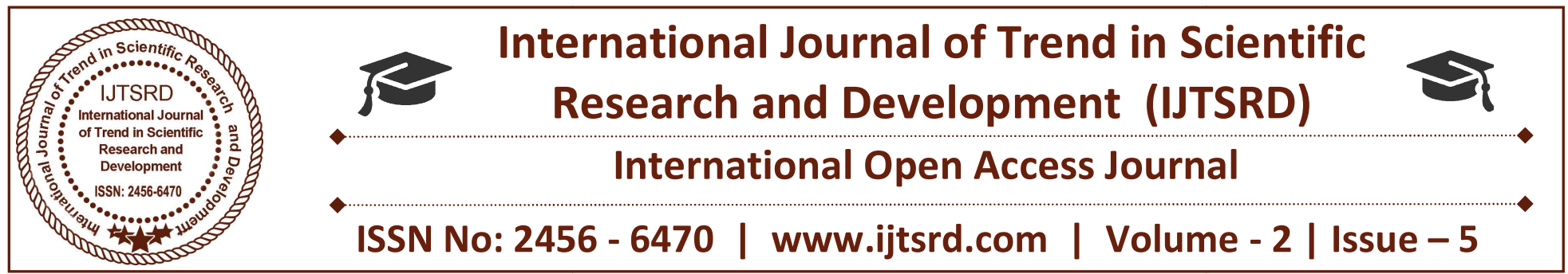

\title{
Analysis on Glaucoma Detection
}

\author{
Sherin. J, Devi Kala Rathinam. D, Santhiya Grace. A \\ PG Scholar \\ Department of Computer Science and Engineering, \\ Sri Krishna College of Engineering and Technology, \\ Coimbatore, Tamil Nadu, India
}

\begin{abstract}
Glaucoma is a disease which leads to a permanent blindness to a person. Many techniques are available in medical industry to detect glaucoma. Some traditional techniques are HRT and COT. But both of them are cost effective and time consuming. These inefficiencies leads to the emergence of automatic computer aided system which can detect glaucoma in less time. Optic disc and optic cup smashes an important role in glaucoma detection. This paper gives an study of various techniques that are used in glaucoma detection using automated systems.
\end{abstract}

Keywords: Glaucoma, optic disk, optic cup, HRT, COT

\section{INTRODUCTION}

Glaucoma is the optic huddle which leads to permanent blindness especially in aged persons. It is very important to detect it in early stages. The medical knacks used by ophthalmologists are Heidelberg retinal tomography (HRT) and Ocular coherence tomography (OCT) to screen glaucoma.To screen glaucoma in time and faster automated computer systems are needed. To screen glaucoma a digital fundus image is used. It consumes less time and gives higher accuracy. As compared to other tangled devices, digital fundus camera is spare economical and brisk. The most common way to detect glaucoma [1] is by finding cup-disk ratio. Optic disk detection is the foremost thing in developing automated diagnosis system. Optic disk is restricted automatically by Principal Component Analysis (PCA) and its shape is detected by Active shape model (ASM). Gaussian Vessel Detector and Tangent Information transform
[4] are used to detect optic disk Centre. Morphological operations and thresholding are commonly used for the detection of optic disk, blood vessels and computational features to analyze glaucoma. During the identification glaucoma results variations in their size, shape, color and depth of optic disk. To give enormous classification in glaucoma wavelet based energy features are applied. Vessel detection and in painting are the two basic steps that are involved in first phase of fundus images. Then the second phase involves CDR calculation. cup disk ratio and ISNT are the two main features used in glaucoma detection. If the cup size increases it affects the neuroretinal rim. fundusimge had an major role in automated segmentation of optic disk and cup disk.

\section{Fundus image:}

It captures the photograph of back of the eye called fundus. some specialized cameras will be there which consists of an microscope attached to a flash enabled camera used in fundus photography. it gives an clear view of the optic and cup disk of our eye retina.

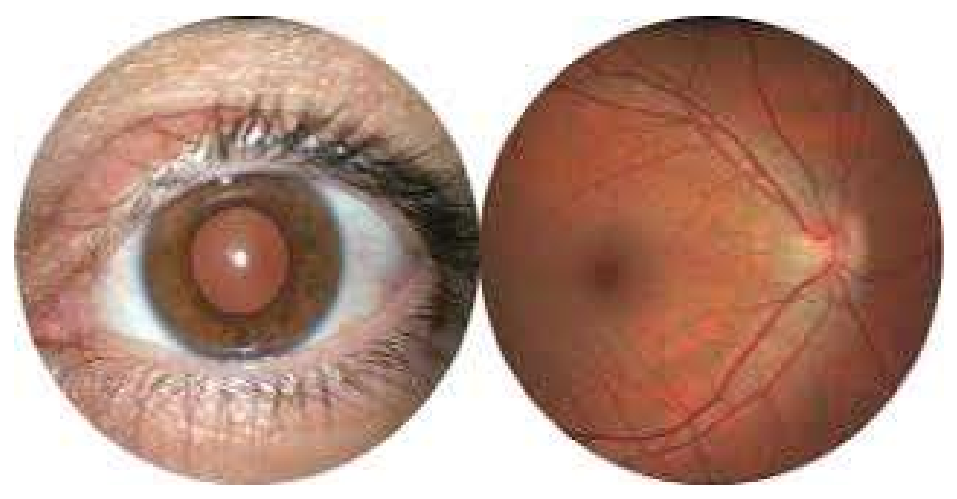




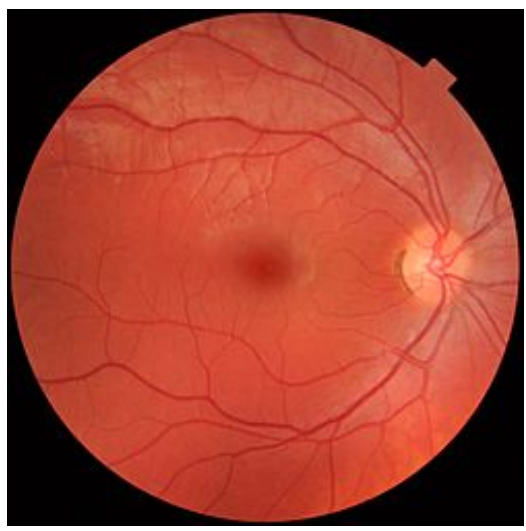

Fig: Fundus retinal image

\section{ANALYSIS}

Regular gluacoma tests includes two common tests. They are as follows :

$>$ Tonometry

$>$ Ophthalmoscopy

$>$ Perimetry

$>$ Gonioscopy

$>$ Pachymetry

\section{A. Tonometry}

It measures the amount of pressure in our eyes.During this test eye drops are used to nub the eye.Then the doctor uses a kind of device which is called a tonometer to measure the inner pressure of our eye.A small amount of pressure is applied to the eye by a tiny equipment.Normal pressure range is $12-22 \mathrm{~mm}$. If the pressure range needs the normal level,gluacoma is diagonised. doctor uses a small evice with a light on the end to light and magnify the optic nerve, If the pressure range is not up to the normal level,it shows the optic nerves unusual.

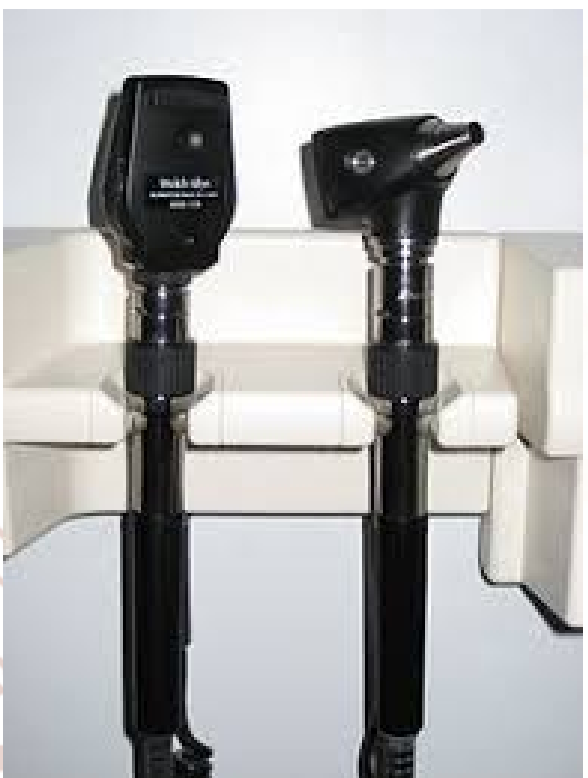

Fig:ophthalmoscopy

\section{Perimetry}

Perimetry gives an visualisable field to produce a map of complete field of vision.It helpsnthe doctor to detect the eye is detected by gluacoma or not.During the test you will be asked to look straight ahead and then light passes, which draw a map of your vision field.

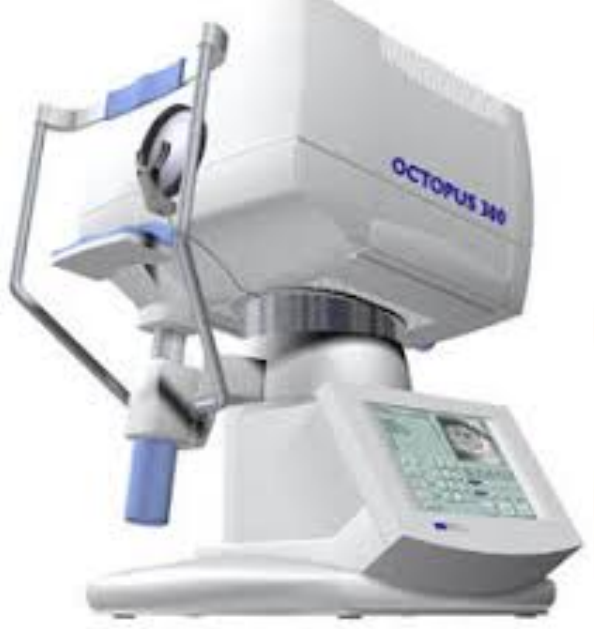

Fig:Perimetry
Fig:Tonometry

\section{B. Ophthalmoscopy}

This procedures examines the optic nerves in your eyes after glaucoma damage.Here also they ues some eye drops to dilate the pupil.Then only the doctor can examine the shape,size of optic nerves in our eyes. The

\section{Gonioscopy}

The diagonostic tests checks the angle between the iris meets the cornea is open and wide or narrow and closed.Here also they uses a eye drops to numb the eye.A hand helded contact lens is placed on the eye.This lens acts as a mirror that shows the doctor if the angle is closed and blocked. 


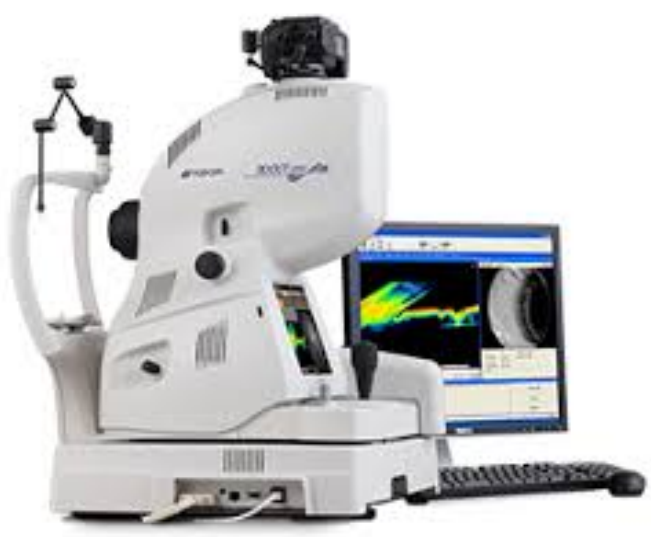

Fig: Gonioscopy machine

\section{REFERENCES}

1. Malay Kishore Dutta, Amit Kumar Mourya, Anushikha Singh, M Parthasarathi, Radim Burget \& Kamil Riha, "Glaucoma Detection by Segmenting the Super Pixels from Fundus Colour Retinal Images" International Conference on Medical Imaging, m- Health \& Emerging Communication System (MEDCOM 2014), pp. 86-90, November 2014.

2. X., Hamaguchi S., Sun Y., Xiao S. "Detect of optic disk centre based on Gaussian vessel detector and tangent information transform" IEEE 2011, 4th international conference on biomedical engineering and informatics (BMEI), Vol.1, pp 250-254, October 2011.

\section{E. Pachymetry :}

This exam is very simple and painless test which measures the thickness of your cornea, a clear window at the front of the eye.A device which is called pachymetre is placed on the front of the eye to measure its thickness. It helps to diagonosis of corneal thickness.It takea s minute to measure both eyes.

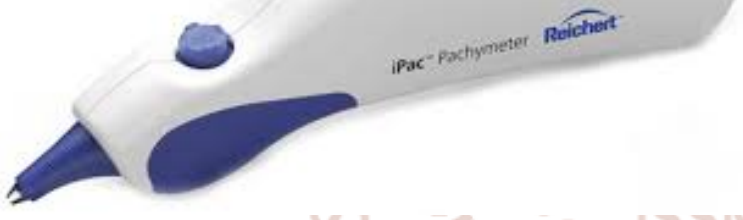

Fig: pachymetry

\section{CONCLUSION}

Glaucoma diagonosis is not very easy,it needs careful evaluation of the optic nerve continuities. The major concern is protecting your sight,so the doctor should looks at many factors before making decisions.Various tests are used to detect gluacoma.Automated machines are mostly used bye the doctors to diagonisis the result due to gluacoma.These technologies gives an faster and accurate results.it reduces the time and increases the performance of the automated conputerised systems.
3. Nayak J., Acharya R., Bhatt P. S., Nakul Shetty, Lim T. C. "Automated diagnosis of Glaucoma using fundus images", Springer science + Business media, LLC 2008. [6] Fengshou Yin, Jiang Liu, Damon Wing Kee Wong, Ngam Meng Tan, Carol Cheung, Manibhaskaran, Tien Yin Wong "Automated segmentation of optic disk and optic cup in fundus images for glaucoma diagnosis", 25th international Symposium on computer based medical system pp.1-6, June 2012.

4. Dua S., Acharya U. R., Chowriappa P., Sree S. V., "Wavelet based energy features for glaucomatous image classification" IEEE transaction on information technology in biomedicine, Vol. 16, no.1,pp 80-87, January 2012.

5. Khan F., Khan S. A., Yasin U. U., Haq I., Qamar U. "Detection of glaucoma using retinal fundus images", The 2013 IEEE Biomedical Engineering International conference (BMEi-CON 2013), pp 1-5, October 2013. [9] Chih-Yin Ho, Tun-Wen Pai, Hau-Teng Chang, Hsin-Yi Chen "An automatic fundus image analysis system for clinical diagnosis of glaucoma", IEEE 2011, International Conferene on Complex, Intelligent and Software intensive systems(CISIS), pp 559564, July 2011. 